\title{
Anthony Barnett
}

Vietnam - China - Kambodscha

Innerkommunistische Auseinandersetzungen und das neue
Vietnam-Problem*

\begin{abstract}
„Die Jugoslawen hatten sich kaum eingerichtet, als Djilas, zwei oder drei Stunden nach seiner Ankunft eine Aufforderung von Stalin erhielt, ihn im Kreml zu besuchen, falls er nicht von der Reise allzu ermüdet sei. Djilas machte sich sofort auf den Weg und wurde von Stalin und Molotow begrïßt. Ohne weitere Vorrede begann Stalin gleich über die albanische Frage zu sprechen: ,Die Regierung der UdSSR erhebt keinerlei Ansprüche irgendwelcher Art auf Albanien. Es steht Jugoslawien jederzeit frei, wann immer es will, Albanien zu schlucken. 'Dieses Wort verdeutlichte Stalin noch durch eine Geste, indem er sich die Finger der rechten Hand leckte.

Djilas war erstaunt über diese Bemerkung und gab zurück: „Aber, Genosse Stalin, hier ist doch nicht die Rede von einem Schlucken Albaniens, sondern von freundschaftlichen Beziehungen zweier verbündeter Länder."

„Na ja, das ist doch ein und dasselbe', bemerkte Molotow."

(aus: Vladimir Dedijer, Tito - Autorisierte Biographie, West-Berlin 1953, S. $302 \mathrm{f}$.)
\end{abstract}

\section{Einleitung}

Die Konflikte zwischen den sozialistischen Staaten Vietnam, China und Kambodscha stellen für Sozialisten eine Krise von großer Tragweite dar und zugleich einen weltpolitischen Wendepunkt. Allgemein gesprochen müssen sich Sozialisten heutzutage mit einer typischen Erscheinung in den Beziehungen zwischen benachbarten kommunistischen Staaten vertraut machen. So wie diese gegenwärtig organisiert sind, brechen zwischen ihnen immer wieder Konflikte aus. Der erste dieser Konflikte ging auf die Herausbildung des Staates zurück, der seine Existenz - als zweiter Staat nach der Sowjetunion - hauptsächlich durch seinen eigenen bewaffneten Kampf gegründet hat, ich meine Jugoslawien. Innerhalb von drei Jahren war Jugoslawien damals auf Betreiben der Sowjetunion isoliert und verfemt. Ich kann an dieser Stelle nicht die ganze Liste der innersozialisitschen Auseinandersetzungen abhandeln, erwähnen muß ich jedoch den zentralen Konflikt, um den sich heute die imperialistische Strategie dreht: den sowjetisch-chinesischen Konflikt. Dergegenwärtige Niedergang der Beziehungen zwischen den kommunistischen Staaten in und am Rande Südostasiens ist nur das letzte Beispiel in einer Reihe, die sich zeitlich beinahe ebenso lang hinzieht, wie $e^{r}$ 'ne Vielfalt sozialistischer Länder gibt.

\footnotetext{
* Dieser Artikel ist die erweiterte Fassung eines Aufsatzes, der im August 1979 in der Zeitschrift der britischen KP Marxism today erschienen ist. Der Autor ist Mitherausgeber der New Left Review, hat intensiy über Südostasien gearbeitet und wird demnächst ein Buch über Kambodscha veröffentlichen.
} 
Wie konnte es dazu kommen? Sozialisten dürfen sich um diese Frage auf keinen Fall herumdrücken, aber sie sollten sich auch nicht mit lauten ,mea culpa"-Klagen nur an die eigene Brust schlagen. Denn diese Konflikte sind zugleich ein Aspekt des Kampfes gegen die weltweite Herrschaft des Kapitalismus. Konflikte zwischen sozialistischen Staaten sind m. a. W. Teil einer imperialistischen Teile-und-Herrsche-Politik. Auf diessem Punkt bestehen heißt keineswegs dem Problem ausweichen. Im Gegenteil: sich angesichts eines Feindes zu zerstreiten, ist nämlich noch schlimmer als unter normalen Umständen ohnehin schon. Auch habe ich zu Beginn auf die eigenartigen Erscheinungsformen hingewiesen, die sich in innersozialistischen Auseinandersetzungen herausgebildet haben, weil in diesen etwas Strukturelles angelegt ist. Die Hammerschläge des Imperialismus haben in den sozialistischen Ländem Staatensysteme entstehen lassen, die militärisch hochgerüstet, hermetisch organisiert und oft diktatorisch regiert sein können (auch wenn sie nicht immer die kriminelle Politik, die pauschalen Säuberungen und die bizarren Kultformen erlebt haben wie die Sowjetunion unter Stalin oder China unter der persönlichen Herrschaft Maos nach 1965). Die Beziehungen, die solche Staaten miteinander unterhalten, sind geheim; demzufolge pflegen auch die kommunistischen Parteien ihre Beziehungen untereinander wie eine besondere Form nationalstaatlicher Diplomatie. Unvermeidlicherweise gewinnt so das nationale Moment die Oberhand über das sozialistische. Unter bestimmten Bedingungen sind natülich Geheimdiplomatie und geduldige Bemühungen um die Beilegung von Differenzen durchaus notwendig. Gerade die vietnamesischen Kommunisten waren in dieser Hinsicht bis zu ihrem Sieg über die US-Truppen außerordentlich geschickt, und ohne solche Methoden wären sie nicht siegreich geblieben. Sie haben aber zugleich ein Bespiel dafür geliefert, wie sozialistische Länder unter dem Zwang zur Verteidigung gegen Angriffe bestimmte Verhaltensformen internalisiert haben, die letztlich ungeheuer zersetzend wirken müssen. Ich werde auf diesen Aspekt zurückkommen, wenn es um den geheimen Charakter der vietnamesischen Beziehungen zu China und Kambodscha geht.

Zunächst jedoch zurück zu meiner Ausgangsfeststellung: die Krise betrifft nicht nur Sozialisten, der Konflikt in. Südostasien stellt darüberhinaus einen ganz besonderen Wendepunkt dar. Die Vereinigung Vietnams, dreißig Jahre nach dem Versuch der Franzosen, das Ergebnis der August-Revolution Ho Chi Minhs wieder rückgängig zu machen, war einer der bedeutendsten Erfolge im Befreiungskampf der Menschheit gegen Ausbeutung und Unterdrückung. Das vietnamesische Volk hat unter Führung seiner kommunistischen Partei - und mit der unersetzlichen materiellen Hilfe, jedoch mit nur zögernder Zustimmung und manchmal sogar gegen den Willen der sozialistischen Länder - die Vorherrschaft der Vereinigten Staaten über die Welt der Nachkriegszeit gebrochen. Heute versuchen die westlichen Medien und die chinesische Presse, und zuweilen ist es schwer, zwischen beiden einen Unterschied auszumachen, die Qualität dieses außerordentlichen Erfolges zu schmälern oder zu triben. Diese Versuche sprechen ihr Urteil über sich selbst.

Der Sieg von 1975 hat mehr Ergebnisse gebracht als nur die Existenz von drei sozialistischen Ländern in Indochina, also von Vietnam, Kambodscha und Laos. Ohne diesen Sieg würde es heute z.B. in Angola keine sozialistische Regierung geben, 
die ihre Macht zu festigen versucht. Aber die Kriege des Jahres 1979 haben den imperialistischen Kräften die Möglichkeit eröffnet, dieses Ergebnis wieder rückgängig $\mathrm{zu}$ machen. Zum ersten gab es eine Kampagne, und es wurde durchaus ernsthaft ins Auge gefaßt, erneut eine direkte Intervention der USA zu legitimieren. Zweitens werden die Vietnamesen verurteilt, um den Krieg der USA noch nachträglich zu rechtfertigen. Genau darum geht es bei der Kampagne, deren Objekt die „boat people" sind: man will den Vietnam-Krieg als einen ,ehrenhaften" hinstellen. Drittens hegt man die Hoffnung, ein ,containment" (Eindämmung) der vietnamesischen Revolution könnte deren humanitäre und demokratische Qualität zerstören, indem sie das Regime unbeliebt werden und seine wirtschaftlichen Anstrengungen scheitern läßt. Der heiße Krieg zwischen den ehemaligen sozialistischen Partnern hat einen neuen kalten Krieg ausgelöst, der gegen den Kommunismus überall gerichtet ist.

Nun darf die Tatsache der Siege von 1975 die sozialistischen Kräfte nicht dazu verleiten, sich über die tatsächlichen Kräfteverhältnisse im Weltmaßstab hinwegzutäuschen. Die Vietnamesen haben die Welt verblüfft, weil sie sich gegen die feindliche Übermacht behauptet haben. Aber diese Übermacht steht nach wie vor Gewehr bei Fuß, und die USA sind - auch wenn ihre übermächtige internationale Position inzwischen gebrochen ist - noch immer eindeutig das mächtigste Land des mächtigsten politischen Blocks. Für Sozialisten im Westen ist es besonders wichtig, diese Tatsache, ohne die kein klares Urteil zu gewinnen ist, zu begreifen und im Auge zu behalten. Heute findet man kaum eine ,seriöse" Zeitung, in der nicht zu lesen wäre, daß der sowjetische Imperialismus mächtiger und aggressiver ist als der Westen, daß der Westen also heute die schwächere von zwei Mächten darstellt, die um die Vorherrschaft kämpfen. Das ist Unsinn. Seit 1975 hat sich die Lage nur insofern geändert, als der sozialistische Block nicht mehr vollständig unterlegen ist.

Die entscheidenden ökonomischen Ziffern sind wahrlich eindeutig. Vergleicht man die Summe aller Bruttoinlandsprodukte der COMECON-Länder mit denen der größten kapitalistischen Länder, so kommt man auf ein Verhältnis von eins zu vier, d.h. die sozialistischen Länder produzieren gerade ein Viertel von dem, was die kapitalistischen Länder (nach den Zahlen der Weltbank) produzieren. Wenn nun das militärische Gleichgewicht eher ausgeglichen ist, so hat die Produktion dieses Gleichgewichts für die sozialistischen Länder weitaus verhängnisvollere Konsequenzen, mußten diese doch ohnehin auf einer viel niedrigeren Basis aufbauen und hatten weit mehr unter militärischen Zerstönngen zu leiden. Darüberhinaus hat das Bedürfnis nach militärischem Gleichgewicht die überkommenen ökonomischen Schwächen der sozialistischen Länder weiter verschärft. Diese Feststellung gilt auch für China - heute vielleicht sogar mehr als für jede andere Geselischaft.

Die Vereinigten Staaten haben bei der Herbeiführung des gegenwärtigen Standes der Feindseligkeiten ganz offensichtlich die Rolle des Anheizers gespielt. Peking hat seine offenen Angriffe auf Hanoi erst gestartet, nachdem Brzezinski im Mai 1978 die chinesische Haptstadt besucht und sich gegen ,regionalen Hegemonismus" (ein auf Vietnam gemüntes Kodewort) gewandt hatte. Als sich in den darauffolgenden Monaten Peking und Hanoi um diplomatische Anerkennung durch Washington 
bemühten, wurden sie von der US-Regierung gegeneinander ausgespielt. Und als Teng Hsiao-ping dann Washington besuchte, erhielt er grünes Licht für einen kurzen Krieg gegen Vietnam. Genauer gesagt: er sprach diese Frage an und erhielt die $\mathrm{Zu}-$ sicherung, daß dadurch die neuen ökonomischen Beziehungen zwischen China und den USA nicht belastet würden. Die Wurzeln dièser Beziehungen gehen sicher bis zu Nixons Besuch in Peking und Moskau im Jahre 1972 zurück, in einer größeren Perspektive sogar bis in die 50er Jahre. Es bereitet auch keinerlei Schwierigkeiten, die spalterische Rolle der USA zu belegen. Aber vor allem muß man dabei verstehen, daß deren Fähigkeit, die Gegensätze zu verschärfen, von ihrer großen ökonomischen Überlegenheit herrïhrt.

Diese einleitenden Bemerkungen sind ein bißchen lang ausgefallen, aber ich glaube, daß ein ausgewogenes Urteil nur im Rahmen des aktuellen Gesamtzusammenhangs möglich ist. Um die Probleme zu klären, die der Konflikt in Südostasien im einzelnen aufwirft, nehmen wir uns jetzt am besten eine Reihe von einzelnen Fragestellungen vor.

\section{Gibt es für den Konflikt einen historischen Vorläufer?}

Ja. Eine offensichtliche Parellele zum Konflikt zwischen China/Vietnam/Kambodscha bietet der Konflikt zwischen Sowjetunion/Jugoslawien/Albanien nach 1945, als die UdSSR die Lage um Albanien.zu verschärfen versuchte, um Jugoslawien in die Knie zu zwingen. Kaum hatten sie 1945 den Faschismus besiegt, da wurden die Jugoslawen 1948 selbst als ,faschistisch“ angeklagt. Zwischen 1945 und 1978 besteht selbst in ideologischer Hinsicht eine enge Parallele, denn die chinesische Presse versucht derzeit, ausgerechnet die Vietnamesen des „Faschismus“ anzuklagen. Jenseits dieser ideologischen Entsprechung hat in den 40er Jahren die Anschuldigung eine Rolle gespielt, Belgrad versuche eine Balkan-Föderation aufzubauen, wobei an einem solchen Projekt gar nichts Unanständiges zu entdecken ist. Eine ähnliche Hysterie pflegen Chinesen heute im Hinblick auf die angeblichen vietnamesischen Bemühungen um eine Indochina-Föderation.

Der Sinn eines solchen Vergleichs liegt darin, besser zu erkennen, daß der primäre Gegensatz heute der zwischen Vietnam und China ist, so wie er damals zwischen Jugoslwien und der Sowjetunion lag; wogegen der Konflikt zwischen den beiden kleineren Staaten diesem größeren Konflikt untergeordnet ist. In beiden Fällen wurde der Versuch der, ,mittleren" Macht, korrekte Beziehungen zu seinem kleineren Nachbarn zu entwickeln, durch die gezielten Aktionen der "Großmacht" kaputtgemacht.

\section{Warum mïndete der Konflikt in Südostasien in einen Krieg?}

Kurz und knapp: weil Kambodscha Vietnam angegriffen hat. In diesem Punkt gibt es überhaupt keinen $\mathbb{Z}$ weifel. Amerikanische Experten, die gegenüber Vietnam aus. 
nahmslos feindlich eingestellt sind und es in die Rolle einer, imperialistischen Macht" drängen wollen, haben durch die Bank zugegeben, daß Kambodscha der Angreifer war. Sie haben Zugang zu den Geheimdienstinformationen der USA, auch müssen sie die Leser ihrer Aufsätze in akademischen Zeitschriften über die grundlegenden Sachverhalte korrekt informieren, wie immer sie diese dann interpretieren. Auf dieser Ebəne - und auch in den Spalten der ",seriösen Presse“ - gilt es als stehende Tatsache, daß Kambodscha Vietnam angegriffen hat (ich beziehe mich dabei auf Experten wie Douglas Pike, Sheldon Simon, Carl Jackson u.a.). Und zwar gab es zwei Phasen der bewaffneten Auseinandersetzungen. Zuerst eine Reihe von Zwischenfällen gleich nach der Befreiung im Jahre 1975. Sie gingen von Kambodscha aus, das die Insel Tho Chu besetzte, und wurden von Vietnam fortgesetzt, das zur Revanche die Insel Wei besetzte. Beigelegt wurden diese Streitigkeiten, gegen Ende des Jahres, als man sich auf einen modus vivendi einigte. 1977 begann dann eine zweite Phase, nachdem sich die Kambodschaner aus der gemeinsamen Grenzkommission zurückgezogen hatten: bewaffnete Auseinandersetzungen gab es vor allem nach dem April 1977, als mehrere kambodschanische Divisionen in die vietnamesische Provinz Tay Ninh einfielen, und erneut im September 1977, als die Kambodschaner - zeitgleich mit der Ankündigung von Pol Pots Reise nach China - an einer 100 Kilometer langen Frontlinie zum Angriff übergingen.

Dies sind nun aber immer noch vietnamesische „Behauptungen“, auch wenn sie von US-Experten akzeptiert werden. Aber wie können Sozialisten sie akzeptieren, wenn die andere Seite solche Tatsachen abstreitet? Der springende Punkt ist, $\mathrm{da} ß$ die andere Seite dies gar nicht tut. Man weiß, daß sich im Sommer 1977 heftige Kämpfe abgespielt haben, aber die Kambodschaner haben den Vietnamesen zu keinem Zeitpunkt zwischen 1976 und September 1977 einen Angriff auf ihr Land vorgeworfen. Infolgedessen müssen die Feindseligkeiten im Frühjahr und Sommer 1977 von Kambodscha ausgegangen sein. Bekannt ist auch, daß im September 1977 schwere Kämpfe stattfanden, die mit der vietnamesischen Gegenoffensive im Dezember endeten. Hanoi gibt letztere nicht offen zu, aber genausowenig leugnet es die Tatsache als solche, die im übrigen allgemein bekannt ist. Folglich müssen wir uns fragen, wer nun recht hat: haben die Vietnamesen im September 1977 angegriffen, wie von den Kambodschanern in ihrer ersten Verlautbarung nach dem Abbruch ihrer diplomatischen Beziehungen zu Vietnam behauptet wurde? Oder haben damals die Kambodschaner in großer Zahl angegrifen und große Zerstörungen angerichtet, wie von Hanoi behauptet wird? Die Antwort darauf ergibt sich aus den „Schwarzen Papieren“. Dabei handelt es sich um das umfangreiche Dokument, das im September 1978 von der Regierung in Phnom Penh veröffentlicht wurde. Das erklärte Ziel dieses Papiers ist es, detailliert die ganzen Verbrechen (wie „Annexionen" $u$.ä.) aufzuzählen, die Vietnam angeblich gegen Kambodscha begangen hat. Bis in die einzelnen Formulierungen hinein trägt es offensichtlich die Handschrift von Pol Pot. Ohne Rücksicht darauf, was sich seine Diplomaten im Jahr zuvor zurechtgebastelt hatten, stellte Pol Pot zumindest in einer Hinsicht die Wahrheit dar: die kambodschanischen "Schwarzen Papiere“ datieren den Beginn der vietnamesischen Angriffe auf Kambodscha im Dezember und nicht im September 
1977. Die Vietnamesen haben sich also schließlich gewaltsam gegen die bedeutenden Aggressionsakte zur Wehr gesetzt, die in den acht vorangegangenen Monaten von April bis September 1977 von Kambodscha ausgegangen waren. Aber das kam die Vietnamesen teuer zu stehen. Denn die Chinesen hetzten die Kambodschaner auf, alle Beziehungen zu Vietnam aufzukündigen und dessen Gegenangriff publik zu machen, so daß Kambodscha als das Opfer erscheinen mußte. Damit gelang es Peking, die Vietnamesen in die Falle zu locken, in die Stalin die Jugoslawen hatte treiben wollen (vgl. das diesem Aufsatz vorangestellte Zitat von Dedijer), denn Pol Pot brauchte damals - als eine nicht so bedeutende politische Figur wie Enver Hodscha - verzweifelt Unterstïtzung und setzte von sich aus auf die militärische Mobilisierung, um sich an der Macht zu halten. Weil aber Hanoi bis zu diesem Zeitpunkt geschwiegen hatte, konnten die Kambodschaner im Dezember 1977 in Peking eine Pressekonferenz abhalten, auf der sie verkündeten, ihr Land werde von Vietnam angegriffen.

Innerhalb weniger Tage zogen die Vietnamesen damals ihre Truppen zurück. Die Kambodschaner feierten dies als einen großen militärischen Sieg, der beweisen sollte, daß sie ihre Nachbarn schlagen konnten. Vietnam antwortete mit seinem Friedensangebot vom 5. Februar 1978 - obwohl die Kambodschaner im Verlauf des vietnamesischen Rückzugs nach Vietnam eingedrungen waren. Der vietnamesische Vorschlag für einen dauerhaften Frieden sah u. a. einen beiderseitigen. Rückzug und eine intemationale Kontrolle der Grenze vor. Die Kambodschaner lehnten dieses Angebot bis April 1978 wiederholt ab. Für mich werden an diesem Sachverhalt zwei Dinge deutlich: erstens wár offenbar das Hauptinteresse der Vietnamesen eine ruhige Grenze. Wenn die „Annexion“6 oder ,Unterwerfung“6 Kambodschas ihr Hauptziel gewesen wäre, hätten sie niemals eine international garantierte Grenze zwischen sich und ihrem Nachbarn vorgeschlagen. Zum zweiten kamen die Hilfeleistungen für Vietnam damals zu einem Drittel aus Peking, und Hanoi hatte deutlich zu erkennen gegeben, daß es im Interesse größtmöglicher Unabhängigkeit Beziehungen zu mehr als einer Seite bevorzugte - kurz zuvor hatte Vietnam ein sowjetisches Angebot abelehnt, dem COMECON beizutreten, und zwar gerade weil dies einen Bruch mit der Volksrepublik China bedeutet hätte. Wenn also China wirklich daran interessiert gewesen wäre, die Integrität Kambodschas zu erhalten, hätte es nicht nur auf Kambodscha einwirken können, das Angebot aus Hanoi anzunehmen, es hätte sogar selbst einen entsprechenden Vorschlag machen müssen. In Wahrheit war es jedoch sein Ziel, Vietnam zu destabilisieren - egal wie hoch die Kosten für das kambodschanische Volk sein würden.

3. Aber warum sollte Kambodscha Vietnam angegriffen haben? Die Annahme ist doch ziemlich unglaubwürdig, es habe eine viel stärkere Macht überfallen.

Zunächst ist, glaube ich, völlig klar, daß die Kambodschaner von Peking Zusicherungen erhalten haben, die fast einer Garantieerklärung gleichkamen. Die Chinesen haben die Feindseligkeiten ermuntert, weil sie sich selbst überschätzten und in inne- 
ren Schwierigkeiten steckten. Aber um etwas zu ermuntern, muß schon etwas da sein: eine Kriegspartei in Phnom Penh, also die Pol Pot-Gruppe. Wie diese Gruppe über die revolutionäre Bewegung Kambodschas hinweg die Macht ergriffen und diese Bewegung zerstört hat, will ich in einer gesonderten Studie darzustellen versuchen. Aber eine erste. Vorstellung von der Instabilität des Regimes, das in Kambodscha nach der Evakuierung der Städte und der totalen Militarisierung des zivilen Lebens durch Arbeitsbrigaden entstanden ist, ergibt sich aus folgender Tatsache: zwischen September 1975 und Mai 1978 hat in Kambodscha alle sechs Monate ein Putschversuch stattgefunden. Eine gewaltige Säuberungswelle folgte lückenlos auf die andere. Einige dieser „Säuberungen“ sind von der Pol Pot-Propaganda als ,vietnamesischer Putsch" ausgegeben worden. Die wichtigste vom April 1977, die den Sturz von zwei hochrangigen politischen Figuren zur Folge hatte, ist nicht so beschrieben worden. Damals scheint vielmehr eine Art chinesischer Putsch stattgefunden zu haben, aus dem Pol Pot als Sieger hervorgegangen ist. Seit diesem Zeitpunkt erfolgten die oben beschriebenen größeren Angriffe auf Vietnam -- und Pol Pot selbst wurde zur gefeierten Persönlichkeit, nicht ohne zuvor (vielen Dank!) die Kommunistische Partei Kambodschas ausgerufen zu haben.

Auch in Afrika war die Welt in jüngster Zeit in zwei Fällen Zeuge von Angriffen kleinerer Staaten auf größere. Somalia überfiel den äthiopischen Ogaden, nachdem es von Saudi Arabien und den USA dazu ermuntert worden war. Uganda griff Tansania an und versuchte damit, seine inneren Spannungen zu lösen, die aus dem Charakter von Idi Amins Regime resultierten. In Kambodscha spielten beide Faktoren -- der Druck von außen wie der von innen - eine Rolle. So unglaublich es klingen mag, der Befund - der sich erst aufgrund einer Analyse des gesamten Revolutionsprozesses in Kambodscha ergibt - lautet ohne jeden Zweifel: Kambodscha hat entlang der gesamten Grenze im Gebiet des Mekong-Deltas Vietnam systematisch angegriffen, und es hat ein vernünftiges Friedensangebot diskussionslos ausgeschlagen.

4. All das zugegeben - warum mußte dann aber Vietnam die Pol Pot-Regierung stürzen? Hätte es sich nicht auf die Verteidigung seiner Grenzen beschränken können, statt Kambodschas ,nationale Souveränität“ zu verletzen?

Meiner Meinung nach verliert eine Nation, wenn sie eine andere militärisch angreift, das Verteidigungsrecht, das auf dem Prinzip der unverletzlichen nationalen Souveränität beruht. Dieses Prinzip kann also sicher nicht als Schild benutzt werden, um einen eigenen Angriff abzudecken. Aber man muß meine Position gar nicht teilen um zu erkennen, daß es sich hier um mehr als um einen Grenzkonflikt handelt. Aufgrund der ökonomischen und sozialen Lage in Südvietnam bedrohten die Angriffe aus Kambodscha den gesamten Prozeß einer friedlichen Vereinigung des Landes und stellten für die Revolution im Süden eine echte Gefährdung dar. Im Hinblick auf den Zeitpunkt der gewaltsamen Übergriffe muß man zwei Dinge im Kopf behalten: Nachdem der Krieg 1978 bekannt geworden war, soll der chinesische 
Botschafter in Laos Berichten zufolge geäußert haben, dies werde ein „langer, langer Krieg“"werden. Und die Vietnamesen haben - anders als die gegenwärtige Hysterie in unserer Presse es weismachen will - das Maß der Gewaltanwendung in ihrem Lande verringert. Die Einrichtung der neuen ökonomischen Zonen, von denen viele nahe der kambodschanischen Grenze liegen, war Teil einer Politik, die von einer unabhängigen UNO-Kommission empfohlen worden war, nachdem sie den Norden und den Süden Vietnams nach dem Krieg bereist hatte, also noch bevor das Land Mitglied der UNO geworden war. Um diese Zonen zu besiedeln, haben die Vietnamesen richtigerweise keine Gewalt gegen die Bevölkerung angewendet. Sie haben unvermeidlicherweise Druck ausgeübt - aber um handfesten Druck handelt es sich auch, wenn etwa ein britischer Schatzkanzler die Arbeitslosigkeit benutzt, um die Löhne niedrig zu halten. Aber zwischen einem solchen politischen Druck einerseits und andererseits Zwangsumsiedlung, befohlener Arbeit und einer Politik, die eine Rückkehr zu normalen Lebensformen bewußt unmöglich macht, liegt eine ganze Welt - und zwar die zwischen Vietnam und Kambodscha. Der vietnamesische Weg war mit einem Handicap behaftet: gerade weil er im wesentlichen ohne Zwang auskam, war er für eine erfolgreiche Rücksiedlung auf friedliche Bedingungen angewiesen. Begleitet von bewaffneten Auseinandersetzungen konnte der Normalisierungsprozeß nicht fortgesetzt werden.

Vielleicht wäre sogar diese Belastung von einer Gesellschaft auszuhalten gewesen, die ihre Spaltung bereits überwunden und eine gewisse innergesellschaftliche Harmonie entwickelt hat. Aber das war im südlichen Teil Vietnams nach 1975 einfach noch nicht der Fall. Die Region von Saigon nach Süden war historisch stets die militanteste des ganzen Landes gewesen, zugleich aber auch das am meisten zersplitterte und unruhigste Gebiet - ganz im Gegensatz zum kompakten und homogenen Norden.Nach ethnischen und kommunalen Gruppen, nach ökonomischen und politischen Kriterien bietet es ein stark fragmentiertes Bild. Es gibt dort zwei große ethnische Volksgruppen: die Khmer und die Hoa (Vietnamesen chinesischer Abstammung), beide zählen mehr als eine Million. Die Khmer wurden von den USA als Rekrutierungsbasis für ihre Spezialtruppen und Konterguerilla-Einheiten benutzt, die auch auf kambodschanischem Boden gegen die Vietnamesen eingesetzt worden waren. Die chinesische Volksgruppe profitierte natürlich ökonomisch vom Krieg und spielte eine bedeutende Rolle in den einschlägigen internationalen Handels- und Nachschubgeschäften, wobei sie von den USA mehr profitierte als von der Befreiungsfront FLN. Jenseits dieser beiden ethnischen Gruppen gibt es drei größere religiöse Gruppierungen und die buddhistische Bewegung; und schließlich auch noch zwei große Sekten: die Hoa Hoa sind eine militante, an das Tausendjährige Reich (der christlichen Offenbarung) glaubende Gruppe, die immer schon anti-kommunistisch war und im südlichen A bschnitt der vietnamesisch-kambodschanischen Grenze siedelte; Teile dieser Gruppe sind ethnisch den Khmer zuzurechnen. Wie die Hoa Hoa hat sich auch die zweite religiöse Gruppe in der Zwischenkriegszeit in Reaktion auf den Druck des französischen Kolonialismus entwickelt. Die Cao Dai haben eine Mischreligion von ausgeprägter Selbständigkeit, ihre Hochburg haben sie ebenfalls im Grenzgebiet, vor allem in der Provinz Tay Ninh, mithin im Bereich der Angriffe vom April 1977. 
Drittens gibt es eine große römisch-katholische Gemeinschaft, die gut organisiert und natürlich auch anti-kommunistisch ist. Fast eine Million von ihnen waren nach der Teilung Vietnams im Jahre 1954 in den Süden gezogen. Dort wurden sie in der Nähe von Saigon angesiedelt, um der Hauptstadt einen gewissen Schutz zu geben; diese katholische Gemeinschaft stellte sich denn auch als soziale Basis für die USgestützten Regierungen des Südens dar. Wie die ethnischen Gruppierungen so entwickelten auch die religiösen Gruppen ihre besonderen politischen und ökonomischen Interessen. In einer Periode sich hinziehender Kämpfe, in der die chinesischen Kaufleute in Reis spekulierten und die Dörfer der Khmer sich der kommunistischen Organisierung widersetzten, ergab sich daraus die mögliche Gefahr einer unkontrollierten gesellschaftlichen Spaltung.

Aber die ökonomische Situation spitzte diese konfliktträchtige Konstellation noch weiter zu. Naturkatastrophen trugen zu einer unzureichenden Ernährungssituation bei, gegen Ende 1978 lagen eine halbe Million Hektar Land brach, das schon gerodet worden war. Für Vietnam war und ist es unbedingt notwendig, dieses Land $\mathrm{zu}$ bebauen und den eigentlichen Kampf gegen die Spätwirkungen der amerikanisclien Entlaubungsaktionen aufzunehmen. Dies aber war unmöglich in einer Zeit aktiver Kämpfe, in der Hunderttausende von Bauern entwurzelt waren und große, extrem fruchtbare Reisanbaugebiete erzwungenermaßen nicht bestellt werden konnten - außer durch Granaten.

Zur Korrektur des Bildes, das bei uns gegenwärtig über Vietnam verbreitet wird, ist auf einen weiteren Punkt hinzuweisen. Im Süden hatten die Partei und die FLN enorme Verluste erlitten. Auch die Kommunisten hatten ihren Teil zum besonders militanten Charakter der Region beigetragen. Die FLN hatte 1963 mindestens 300000 Mitglieder umfaßt. Aber die Bewegung und ihre Kader waren beinahe vollständig ausgelöscht worden, an vielen Orten bis zu 96 oder $97 \%$. Nur die eng. sten Kader hatten im Untergrund überlebt. Das US-amerikanische Phönix-Programm hatte darüberhinaus Zehntausende von Dorfaltesten eliminiert; viele von ihnen waren keine Parteikader gewesen, sondern geachtete Einzelpersonen, die ihre Gemeinden seit der Befreiung durch die FLN (vor 1965) verwaltet hatten. Zu der inneren Zerrissenheit des Südens kam also die enorme Schwierigkeit hinzu, den Kriegsfolgen in einer politischen Konstellation gegenüberzustehen, in der auf lokaler Ebene die Sieger weit höhere Verluste erlitten hatten als die Verlierer.

Damit komme ich zur letzten Gruppe, zu den über eine Million südvietnamesischen Regierungstruppen, den Zehntausenden von Polizisten und Beamten des Thieu-Regimes, ganz zu schweigen von den vielen anderen direkt bei US-Institutionen Beschäftigten, die durch den Sieg von 1975 ihren $\mathbb{J}$ ob eingebüß hatten. Die Komnunisten taten und tun alles erdenkliche, um diese Menschen für sich zu gewinnen - oder wenigstens dazu zu bringen, die Regierung in Hanoi zu respektieren und die Gesetze zu befolgen, sowie ihren Lebensunterhalt unter Bedingungen zu verdienen, die nicht mehr yom reichsten $\mathbb{L}$ and der Welt subventioniert werden. Vor dem Hintergrund eines sich hinziehenden, von den kambodschanischen Kommunisten verursachten Krieges war es aber nahezu unvorstellbar, daß sich eine solche neue Loyalität herausbildete. 


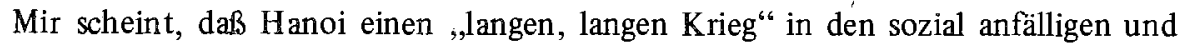
wirtschaftlich produktiven Grenzgebieten zu Kambodscha unter keinen Umständen hätte hinnehmen können. Aus vie tnamesischer Sicht war ein Krieg in den entlegenen Gebieten des kambodschanischen Berglandes offensichtlich vorteilhafter. Militärisch würłe das zwar größere Verluste an Truppen und Ausrüstungen und ökonomisch eine größere Belastung bedeuten. Aber politisch und sozial konnte man einen solchen Krieg verkraften, während eine Defensivoperation, bei der den kambodschanischen Truppen und ihren chinesischen Beratern (davon gab es mindestens 10000 ) die Initiative überlassen blieb, auf keinen Fall hingenommen werden konnte. Um im Interesse seiner eigenen Verteidigung zurückzuschlagen, mußte Vie tnam also bis zur letzten Konsequenz gehen.

Daraus ergibt sich ein letzter Punkt, auf den ich hinweisen muß. Das Pol PotRegime war das schrecklichste, das jemals im Namen des Sozialismus errichtet wurde, und ich befürchte, daß die Gesamtbilanz dieses Regimes schlimmer aussehen wird, als man es sich heute offenbar vorstellen kann. Aber das war nicht der Grund für den Sturz dieses Regimes durch die Vietnamesen. Vielleicht war es der letzte Grund, der die Aktion nötig machte, aber das primäre Motiv war für Hanoi die Verteidigung seines nationalen Entwicklungsprozesses. Aus diesem Grund hat es eine internationale Überwachung der entmilitarisierten Grenze vorgeschlagen, die sicherlich für alle vorhersehbare Zukunft jegliche vietnamesische Vorherrschaft über Kambodscha verhindert hätte. Vietnams primäres Motiv und Ziel war es weder, durch unstatthafte Methoden oder durch Gewalt seine Hegemonie über ganz Indochina abzusichern, noch in einem selbstlosen Akt gegenüber dem kambodschanischen Volk und der ganzen Welt der Pol Pot-Regierung ein Ende zu setzen. Es war eine defensive Operation, die der Lösung desselben Problems galt, das sich für die Führung in Hanoi eine ganze Generation lang so unnachgiebig gestellt hat: der Wiedervereinigung des eigenen Landes.

5. Das heißt also, daß Pol Pot nicht nur eine außergewöhnliche Innenpolitik, sondern auch eine Aggressionspolitik nach außen verfolgt hat. Wie kann man aber ein solches Regime als „sozialistisch" bezeichnen? Wie ist uberhaupt die kambodschanische Gesellschaft unter Pol Pot zu kennzeichnen?

In seinen Reden hat Pol Pot den Anspruch vertreten, den Sozialismus in Kambodscha mit einem Schlag verwirklicht zu haben; er sprach von der Zerschlagung der Bourgeoisie und rechtfertigte die Abschaffung des Geldes mit antikapitalistischen Begriffen. Insofern ist nicht einzusehen, wie man das ,Demokratische Kampuchea“ als Variante eines kapitalistischen Staates, also etwa als faschistisch, charakterisieren kann. Dieser Ausdruck ist von mehreren Kommentatoren, auch von vietnamesischen, benutzt worden. Hinsichtlich des Rassismus, der Pogrome und des Expansionismus und Militarismus trifft er scheinbar zu, konzeptionell läuft er jedoch auf dem falschen Gleis. Sobald man ein bißchen genauer nachdenkt, wirft der Versuch, das Pol Pot-Regime unter einen Kapitalismus- oder Faschismus-Begriff zu fassen, 
mehr Probleme auf als er löst. Das ganze mag einem kurzfristigen Ziel dienen, indem es Kambodscha ins andere Lager schiebt, aber es beantwortet nicht die Frage: wie konnte Pol Pot überhaupt die Macht erringen? Wie konnte ein „Faschist“" an die Spitze einer nationalen Befreiungsbewegung gelangen? Warum haben Länder wie Kuba zu einer faschistischen Führung brüderliche Beziehungen angestrebt? Bahro benutzt den Begriff „,gegenwärtig existierender Sozialismus“ - nun, Pol Pots Kambodscha hat aktuell in der Tat existiert und seine Führer betrachteten sich selbst als marxistische Revolutionäre. Ich glaube, daß jeder von uns hier im Westen ein paar politische Aktivisten nennen könnte, die sich überzeugt und heroisch einsetzen, große Opfer bringen, der marxistischen Linken angehören - und dennoch die letzten wären, die man jemals in staatlichen Machtpositionen sehen wollte. In Kambodscha hat eine solche Gruppe die Führung der ganzen kommunistischen Bewegung errungen.

Der Begriff ,stalinistisch" hat heutzutage in der Regel jede Bedeutung eingebüßt, inzwischen wird er sogar auf bürokratische Manipulationen in der Arbeiterbewegung angewandt oder auf die elementaren Industrialisierungsanstrengungen in rückständigen Ländern. In meiner Studie über Kambodscha versuche ich sehr kurz und vorläufig, dem Begriff eine knappe und spezifische Bedeutung zu geben, die durch folgende Merkmale umrissen werden kann: persönliche Diktatur und Parteisäuberungen (mit terroristischen Mitteln); forcierte Industrialisierung und repressive Lenkung der Volksmassen; Isolierung des Landes und Nationalkult. Stalinismus wäre demnach in einer kurzen Formel eine diktatorische nationale Entwicklung mit zentralisierter Ökonomie. Ich vertrete die Auffassung, daß Kambodscha unter Pol Pot der Unterfall einer solchen Gesellschaft gewesen ist. In der gebotenen Kürze kann ich hier nur auf die ökonomische Ebene näher eingehen.

Das „demokratische Kampuchea" bot nach der völligen Entvölkerung der Städ te das Bild einer ländlich-bäuerlichen Gesellschaft, die sowohl das westliche wie das sowjetische Modernisierungs-Vorbild ablehnte. Seine Verteidiger meinen manchmal sogar, es habe sich wieder enger auf „die Natur" bezogen. So sympathisch mir die Hoffnungen sind, die sich hinter dieser. Ansicht verbargen, so sehr haben sie im Falle von Kambodscha getrogen. In einem Land, in dem es historisch - außer in der Provinz Battambang - fast keinen Großgrundbesitz gegeben hat, wurde das gesamte Ackerland kollektiviert. Das Regime war auf diese Errungenschaft schrecklich stolz und hat sie in seiner Verfassung festgeschrieben. Die Bauern wurden im wahrsten Sinne des Wortes enteignet und unter strengen Bedingungen zum Arbeitseinsatz gezwungen, zuletzt wurde sogar das Essen reglementiert. Bei der Organisation umfassender Erdarbeiten wurden Arbeitsarmeen von bis zu 20000 Menschen von einer Baustelle zur anderen dirigiert; gearbeitet wurde für kleinste Rationen, deren Zuteilūng auf individueller Grundlage erfolgte (nur wer soundsoviel Kubikmeter Erde wegschaffte, erhielt seine Reisration!). Die ganze kambodschanische Landwirtschaft wurde zu einer einzigen ungeheuren und kruden Plantagen-Ökonomie. Um die Produktion zu erhöhen, wurden Wasserbauwerke aus dem Boden gestampft, die eine primitive Form ländlicher Kapitalakkumulation darstellten. Kurz gesagt: Kambodscha erlebte die zwangsweise Industrialisienung seiner Landwirtschaft. Der Reis, der 
auf diese Weise expropriiert wurde - man kann ihn kaum einen „Überschuß“(surplus) nennen, denn die Bevölkerung blieb dabei hungrig - wurde auf dem Weltmarkt verkauft, nach China verschifft oder von der Armee verbraucht. Daß es sich bei dem ganzen Unternehmen um eine Agrarindustrie ohne Maschinen handelte, ändert nichts an der Grund tatsache, machte aber die menschlichen Leiden nur umso schrecklicher.

Bleibt die Frage, warum die Pol Pot-Gruppe diese unerhörte Arbeitsanstrengung angeordnet und zugleich einen Krieg angefangen hat. Das eine war irrational genug, beides gleichzeitig zu unternehmen war ein Wahnsinn, der selbst in der Geschichte Europas seinesgleichen sucht. Stalin etwa hat wohl seine berühmte dreifache Revolution durchgezogen (Zwangskollektivierung, ,große Säuberung“, Industrialisierung um jeden Preis), aber dennoch in dieser Zeit die Sowjetmacht konsolidiert, indem er sich beträchtlichen Rückhalt in der Zivilbevölkerung verschaffte und sich auf internationaler Ebene defensiv verhielt. Anders als Stalin leitete Pol Pot jedoch die kambodschanische Revolution gleichsam hinter verschlossenen Türen. Das machte seine Politik aggressiver, denn obwohl er die Partei führte, war er dennoch von der Befreiungsbewegung isoliert, die diese Partei organisiert hatte - eine Isolierung, für die die Geheimhaltungspolitik der Partei und seiner eigenen Person das stärkste Symbol waren. (Der Maoismus vorl Pol Pot und seinen Anhängern hat darüberhinaus zu einem militanten Nationalismus beigetragen, aber das wäre ein Thema für sich).

An dem ganzen Regime war etwas ,pathologisches“. Dies kann ganz unbefangęn festgestellt werden. Auf einen Zug der Pol Pot-Herrschaft war seine Gruppe geradezu besessen stolz: auf ihre angeblich totale und vollständige Unabhängigkeit. So bestritt man etwa, irgendwelche Hilfe von Vietnam erhalten zu haben, wo doch nur zu bekannt war, daß vietnamesische Truppen die erste Offensive Lon Nols gegen die kambodschanische Befreiungsfront zurückgeschlagen hatten. Ich will damit nicht sagen, die Kambodschaner hätten keine eigenständige Revolution zustande gebracht. Aber Tatsache ist, daß sie dabei entscheidende Hilfe und Beistand erhalten und daß viele Vietnamesen auf dem kambodschanischen Schlachtfeld ihr Leben gelassen haben. Aber die kambodschanische Führung war unfähig, dies auch nur am Rande zu erwähnen. Als sie sich später mit ihrer absoluten und vollständigen Unabhängigkeit brüstete, war sie viel umfassender von einem einzigen Land (nämlich China) abhängig, als es Sihanouk jemals gewesen war, dessen "Semi-Unabhängigkeit' ${ }^{*}$ Pol Pot verflucht und verachtet hat. Die „Luigen“", die in diesem Punkt zu verzeichnen sind, unterscheiden sich in einer wichtigen Hinsicht von den kalkulierten und taktischen Lïgen, die Hanoi für notwendig gehalten hat. Wenn jemand wirklich glaubt, ein Riese zu sein, wo er doch nur ein ganz gewöhnlicher Mensch ist, so bedeutet dies, daß er den Bezug zur Realität eingebüßt hat - und dies ist eine pathologische Erscheinung. Ich will damit nicht nahelegen, daß Pol Pot verrückt gewesen und deshalb nicht mehr zu begreifen sei. Ich will im Gegenteil klarstellen, daß wir in diesem Fall versuchen müssen, eine irrationale Erscheinung zu begreifen. Die materielle Grundlage für diesen Realitätsverlust war letztlich die totale Isolierung der Pol Pot-Gruppe vom kambodschanischen Volk - eine lsolierung, die im Moment des Sieges über den US- 
Imperialismus komplett wurde, als das Regime die zwangsweise Evakuierung der Städte durchsetzte.

6. Aber wenn der Fall so eindeutig liegt, warum starteten die Vietnamesen dann eine geheime Invasion nach Kambodscha, die sie nach außen hin nicht zugaben?

Wie eingangs angedeutet wirft dieses Thema ein Schlaglicht auf einen der Gründe, warum sozialistische Staaten gegenwärtig die Neigung an den Tag legen, miteinander in Konflikt zu geraten. Die Geheimhaltungspolitik Hanois lief in zwei Phasen ab und auf beide reagierten die westlichen Medien mit systematisch entstellenden Berichten. In der ersten Phase haben die Vietnamesen wie gesagt verschwiegen, daß sie unverhüllt angegriffen worden waren. Dadurch haben sie die Gelegenheit für den Beweis verpaßt, daß sie ganz eindeutig Opfer einer Aggression waren, die in viel größerem Maßstab abgelaufen ist als die vorangegangenen Übergriffe der Roten Khmer auf Dörfer in Thailand. Warum haben die Vietnamesen nicht Alarm geschlagen? Zunächst, weil sie keine unwiderrufliche Auseinandersetzung mit China provozieren bzw. Peking und der Pol Pot-Gruppe nicht durch einen offenen Angriff auf Kambodscha in die Hände spielen wollten. Dieses Vorgehen erschien der Führung in Hanoi - abgesehen davon, daß keine politische Gruppe eine Entwicklung gern außer Kontrolle geraten läßt - aus zwei Gründen als vernünftig. Zum einen war ihnen eine solche Methode von Ho Chi Minh beigebracht worden. Die Vietnamesen hatten schon sehr schwierige Differenzen mit der Sowjetunion und China einfach dadurch überwunden, daß sie sich ruhig verhielten, daß sie mit taktischen Mitteln die Situation $\mathrm{zu}$ verändern suchten, und daß sie schließlich auf einer pragmatischen Ebene eine erneute Einigung erzielten. Im Vergleich dazu waren die polemischen Auseinandersetzungen zwischen Moskau und Belgrad bzw. zwischen Peking und Moskau objektive Lehrstücke für den Verfall der Verkehrsformen innerhalb der sozialistischen und internationalen Beziehungen gewesen.

In Vietnam wußte praktisch jeder, daß die Angriffe aus dem einen oder anderen Grund von einem Regime ausgingen, das die Städte entvölkert, das Geld abgeschafft u.v.m. A ber offiziell wußte es niemand. Und so wußten es auch die Anhänger des indochinesischen Befreiungskrieges im Westen oder in der Dritten Welt nicht, und es gab auch keine Rundfunksendungen an das kambodschanische Volk mit der Aufforderung, eine weitere Eskalation des Krieges zu verhindern. Indem Hanoi schwieg, sorgte es dafür, daß die Opposition gegen Pol Pot auf die diplomatische oder die verdeckte oder die konspirative Ebene beschränkt blieb. Zu keiner Zeit übte man offenen politischen Druck aus, der Unterstützung für die Vietnamesen hätte mobilisieren können.

Aber es gab noch einen weiteren Grund für Hanois Zurückhaltung, die nicht einfach nur eine Fortsetzung der Politik Ho Chi Minhs war. Nach den Kämpfen von 1975 war eine Art Windstille eingetreten und die Möglichkeit einer Einigung hatte sich abgezeichnet. Sowohl die Führung in Phnom Penh als auch die in Peking waren $\mathrm{zu}$ dieser Zeit ausgesprochen instabil. Diese Instabilität drängte sie dazu, sich mit 
Hanoi anzulegen, war doch in beiden Hauptstädten keine Gruppe sicher genug an der Macht und damit selbstsicher genug, um sich mit Hanoi friedlich zu einigen. Eine solche zerstrittene Situation bedeutete andererseits Unsicherheit und für Hanoi mithin die Möglichkeit, einen ernsten Konflikt über einen erneuten internen Kurswechsel in den Nachbarländern abzuwenden. Deshalb war in dieser Situation Schweigen Gold. In Hanoi wurde wahrscheinlich angenommen, eine andere Haltung würde alle Fraktionen in Peking in eine feindliche Haltung hineindrängen. Der entscheidende Moment war in dieser Hinsicht höchstwahrscheinlich der Besuch des vietnamesischen Regierungschefs Le Duan in Peking im November 1977, kurz nach dem Besuch Pol Pots. Obwohl dieser Besuch offenbar in freundlicher Atmosphäre verlief, drohte Peking nur einen Tag nach Le Duans Abreise ganz unvermittelt mit einem Krieg wegen der (vietnamesischen) Spratley-Inseln. Spätestens damals muß man in Hanoi gemerkt haben, daß so gut wie alle politischen Handlungsmöglichkeiten durch Peking verstellt waren. Dennoch - die Hoffnung, mit China eine Art Aussöhnung zu erreichen, war nicht völlig grundlos gewesen, und Vietnam hat bis hin zum stillschweigenden Hinnehmen von Angriffen alles für dieses Ziel getan. Aber im Rückblick war das ein, wenn auch verständlicher, Fehler.

Nachdem schließlich der Konflikt Ende Dezember 1977 öffentlich bekannt geworden war, gab Hanoi eine lange und glaubwürdige Erklärung heraus. Man lud Reporter ein. In der Far Eastern Economic Review erschien eine Titelgeschichte, in der die kambodschanischen Angriffe auf die vietnamesische Provinz Ha Tien im einzelnen aufgezählt wurden. Aber die westlichen Medien versuchten erst gar nicht, über die Fakten dieses Falles zu berichten. Man beschränkte sich darauf, das Schauspiel zu genießen, das von zwei kommunistischen Verbündeten bei ihrem Kampf gegeneinander geboten wurde. Nachdem das Schweigen gebrochen war, wurde die Wahrheit durch die Art und Weise verzerrt, in der über dieses Thema berichtet wurde. Aber für diese Verzerrung können wohl kaum die Vietnamesen verantwortlich gemacht werden.

$\mathrm{Da} \mathfrak{3}$ Hanoi seine Invasion unternommen und zugleich behauptet hat, in Kambodscha habe ein „Aufstand“ stattgefunden, den man lediglich unterstütze, hat dagegen anders gelagerte Gründe. Auch in dieser Hinsicht finden wir für eine solche Politik erfolgreiche historische Vorbilder. Als vor 1975 vietnamesische Truppen in Laos bzw. in den befreiten Gebieten Kambodschas standen, hat Hanoi dies auch bestritten. Jeder wußte darüber Bescheid, aber das Faktum offen zuzugeben, wäre eindeutig weniger , wahr ${ }^{66}$ gewesen als die vietnamesische Haltung des Abstreitens. Und zwar deshalb, weil die Behauptung von den Amerikanern ausging, die damit weiszumachen versuchten, die Vietnamesen hätten ihr Land, überfallen", obwohl die eigentliche Aggression eindeutig in der Verletzung der Neutralität von Laos oder Kambodscha durch die USA und in der Liquidierung des Genfer Abkommens bestand. Hätte man sich damals über die vietnamesischen „Verstöße " ereifert, hätte das praktisch geheißen, die viel größeren der USA stillschweigend gutzuheißen. Und nebenbei standen die vietnamesischen Truppen auch nicht in Vientiane oder Phnom Penh. Insgesamt hat es sich für Hanoi also ausgezahlt, die Souveränität seiner Nachbarn - übrigens mit Einverständnis der dortigen kommunistischen Parteien, und im 
Fall von Kambodscha auch mit geheimer Zustimmung von Sihanouk - ,anzutasten“ und dies zugleich abzustreiten.

Als dieselbe Methode der Geheimhaltung nunmehr auf Kambodscha ausgedehnt wurde, trat ein Bumerang-Effekt ein. Die Invasion eines ganzen Landes, die man nicht einmal zugibt, muß als unglaublich heimtückisch erscheinen. Insbesondere die Länder der Dritten Welt wurden durch diese Form einer unehrlichen Politik vor den Kopf gestoßen und Hanoi hätte sicher besser daran getan, offen und ehrlich vorzugehen. Warum hat man das nicht getan? Einmal, weil die vietnamesischen Kommunisten die Vorstellung, ihr Land sei nur ,begrenzt souverän ${ }^{6}$, nicht akzeptieren; schließlich haben sie dreißig Jahre gekämpft, um erst einmal ihre eigene Souveränität zu erringen. Das letzte, was sie zulassen würden, wäre eine Politik internationaler Beziehungen, die die Chinesen gegen Vietnam ausnutzen könnten. Weiterhin habe ich den Eindruck, daß sie hofften, andere Länder der Dritten Welt würden ihre diplomatischen Dementis hinnehmen, zugleich aber ihre militärische Zwangslage verstehen. Zum dritten waren sie nicht in der Lage, die Weltöffentlichkeit auf ihre Gegenmaßnahmen vorzubereiten, denn vom militärischen Standpunkt aus waren sie auf das Überraschungsmoment absolut angewiesen. Wenn sie die Chinesen im Voraus gewarnt hätten, wären die Folgen unter Umständen weitaus gravierender gewesen. (In der Tat gab es ein Überraschungsmoment: westliche Nachrichtendienste und wahrscheinlich auch Peking haben lediglich eine begrenzte Invasion bis zum Mekong-Ufer erwartet, die zwar zur Evakuierung von Phnom Penh geführt, sich aber nicht auf ganz Kambodscha erstreckt hätte. Nach dieser Annahme hätte die nationale Befreiungsfront dann Truppen rekrutiert und den Kampf allein fortgesetzt.)

Im Rückblick wäre es zweifellos besser gewesen, wenn die Vietnamesen die Evakuierung der Städte und den Charakter des kambodschanischen Regimes kritisiert und die Kämpfe von 1977 offen angezeigt hätte. Aber es gibt eben heutzutage zwischen kommunistischen Ländern keinerlei Zwischenstufen zwischen feindseligen Auseinandersetzungen und der Vertraulichkeit diplomatischer Verhandlungen über strittige Punkte. Das Recht, konstruktive Kritik zu üben - mit allen notwendigen Folgen für die innere Demokratie - ist in der sozialistischen Welt noch nicht entwickelt. Zum Teil liegt das natürlich am Fehlen einer Presse, die der proletarischen Tradition verpflichtet, zugleich aber vom Staat unabhängig ist, also einer sozialistischen Presse, die nicht im Rahmen der engen Grenzen staatlicher Politik funktioniert. Das ist gerade in Südostasien ein Punkt von größter Bedeutung, weil dort ein neues Gefuge von Staaten entstanden ist, das durch ein Dreieck von Freundschafts. verträgen miteinander verbunden ist. Hier muß eine Sprache wechselseitiger Verständigung gefunden werden, die den Bedürnissen der Parteien in allen drei Ländem entspricht. Im Augenblick ist es jedoch so, daß die Präsenz einer Volksrepublik China, die die Differenzen anzustacheln versucht, diese drei Länder dazu zwingen wird, ihre ganz natürlichen Konflikte unter der Decke zu halten, so daß sie, anstatt aufgelöst und bereinigt żu werden, unter der Oberfläche weiterwirken, unterdrückt werden oder gar einen unnatürlichen bzw. antagonistischen Ausdruck finden.

Abschließend muß festgehalten werden, daß es gegen Pol Pot in der Tat, wie von Hanoi behauptet, Widerstand gegeben hat. Hingegen glaube ich nicht, daß die 
Vietnamesen einmarschiert wären, wenn sie mit dem Widerstand des Volkes gegen sich selbst hätten rechnen müssen. Den hat es dann auch nicht gegeben. Dagegen gab es Widerstand und Opposition gegen Pol Pot, und das war auch die Grundlage für die rasche Isolierung der Roten Khmer, die bisher bemerkenswert schnell vonstatten ging, wenn man bedenkt, wie groß die kambodschanische Armee gewesen ist, wieviel Vorbereitungszeit sie hatte, und wie viele Vorteile sie in einem Guerillakrieg auf kambodschanischem Gebiet genießt. Aber man darf auch nicht vergessen, daß die Elite der kambodschanischen kommunistischen Bewegung durch die erwähnten Säuberungen liquidiert worden ist, nachdem es ihr nicht gelungen war, ihre Opposition gegen Pol Pot auf einen gemeinsamen Nenner zu bringen.

\section{Hat die Sowjetunion bei all diesen Entwicklungen keine Rolle gespielt?}

In einer Zeit, da im Westen zu diesem Thema nichts Unverzerrtes zu hören ist, muß man mit seinem eigenen Urteil sehr abwägend und genau sein. Zunächst hat die Sowjetunion Vietnam mit sehr großer militärischer und ökonomischer Hilfe unterstützt. Sie vor allem hat die Isolierung der vietnamesischen Revolution verhindert. Jegliche Kritik hat zunächst von dieser Tatsache auszugehen, für die wir alle sehr dankbar sein sollten. Gleichzeitig hat aber die UdSSR von dem Konflikt profitiert, denn sie hat damit einen Hebel gegen China gewonnen: dank Vietnam werden die beiden Länder jetzt vielleicht wieder ernsthaft miteinander verhandeln. Die sowjetische Unterstützung ist also durchaus nicht uneigennützig. Dafür ist die Tatsache ein klares Anzeichen, daß für Kambodscha nicht genügend Hilfe geleistet wird. Ich habe den Eindruck, daß die Vietnamesen den Sowjets nicht gestattet haben, in Kambodscha in größerem Umfang Einfluß zu gewinnen, weil Hanoi ein Abkommen mit China erreichen will und die politische Entwicklung in Kambodscha dabei offensichtlich von großer Bedeutung ist. Ohne ein politisches Tauschgeschäft hat die UdSSR womöglich die Hilfe zurückgehalten, die so notwendig gebraucht wird (ich möchte betonen, daß diese These nicht klar belegbar ist); eine Hilfe, die dem Volk dort ganz uneigennützig gegeben werden muß und die der Sowjetunion große Wertschätzung verschaffen würde - aber keinen stärkeren Druck auf Peking ermöglichen und den sowjetischen Einfluß auf Hanoi eher vermindern würde.

Die aktuellste kritische Frage ist die, wie Vietnam zur Sowjetunion steht. Ganz zweifellos haben die Vietnamesen das Gefühl, daß die Chinesen sie in eine Lage drängen wollen, die sie von Moskau abhängig machen würde. Die Vietnamesen haben den Krieg gegen China mit drei Zielen geführt. In der Reihenfolge ihrer Wichtigkeit waren dies:

sie wollten die chinesischen Truppen festnageln und deren militärischen Erfolg verhindern; dieses Ziel wurde erreicht;

- sie wollten ihre Positionen in Kambodscha halten und diese durch die chinesische Einmischung nicht gefährden lassen; auch in dieser Hinsicht waren sie erfolgreich;

- $\quad$ sie wollten die sowjetische Beteiligung auf ein Mindestmaß beschränken; in diesem Punkt waren sie relativ erfolgreich. 
(Es scheint übrigens ganz sicher $\mathrm{zu}$ sein, daß es direkte bilaterale Verhandlungen über Vietnam zwischen Moskau und Peking gegeben hat, was Hanoi natürlich nicht willkommen war.) Einer der Gründe, warum das vietnamesische Oberkommando auf die chinesische Invasion lediglich auf der kleinsten Flamme reagiert hat, die gerade zum Erfolg ausreichte, lag darin, daß man die Sowjetunion nicht hineinziehen wollte.

Die Chinesen und die USA wollen andererseits beide - aus sehr verschiedenen und letztlich vielleicht kontroversen Gründen - Vietnam in Abhängigkeit von der Sowjetunion hineindrängen. Sie wollen die Beziehungen Vietnams zu anderen Ländern kappen und Hilfe jedweder Art für das Land verhindern. Da ich diese Erörterung mit dem Hinweis auf Jugoslawien begonnen habe, kann ich vielleicht auch mit einem Hinweis auf dieses Land schließen. Mir kommt es wie eine besonders bittere Paradoxie vor, daß eines der führenden Länder der blockfreien Bewegung eine der chinesischen ähnliche Position bezieht und Vietnam angreift. Vietnams politische Bilanz erweckt wahrlich nicht den Eindruck, als neige das Land zu einem SatellitenStatus! In Südostasien hat eine ganze Reihe bedeutender kommunistischer Revolutionen stattgefunden, und zwar auf der Grundlage von internationaler Solidarität, von mehrseitigen Beziehungen und von sozialistischer Unabhängigkeit. Was könnte es für die Dritte Welt bringen, wenn Vietnam in eine Lage gedrängt würde, in der es nur noch von den orthodoxen sozialistischen Ländern Unterstützung erfährt? Nicht die Vietnamesen hören auf blockfrei zu sein, sondern: Vietnam wird, vor allem von China, in Richtung der Sowjetunion gedrängt, und in einer solchen Situation muß3 man sogar noch froh sein, daß die Sowjetunion da ist. Aber über China hinaus spielen offensichtlich Länder wie Jugoslawien dasselbe Spiel und hindern Vietnam daran, seine eigene, deutlich eigenständige und kreative Rolle zu spielen, indem sie ihm Kalte-Kriegs-Definitionen aufzwingen -- aus dem einzigen Grund, weil Hanoi sich weigert, sich Peking zu unterwerfen (und wer sollte gerade dafür mehr Verständnis haben können als Jugoslawien?). Die Rolle Belgrads müißte es doch wahrlich sein, eine weitere Polarisierung zu verhindern, statt sie voranzutreiben, wie es im Augenblick den Anschein hat.

Es ist durchaus verständlich, daß europäische Sozialisten die Sowjetunion wegen ihrer Invasion der CSSR mit Mißtrauen beobachten, wenngleich dieses Mißtrauen heutzutagen in einer Art und Weise, ausgenutzt wird, die man prinzipienlos nennen könnte. Aber es ist grotesk, diese regionale Perspektive auf den Rest der Welt zu projizieren. Grotesk deshalb, weil dadurch genau die Art bürokratischer, eigensüchtiger und defensiver Mentalität ausgebrütet wird, gegen die man angeblich etwas hat. Wenn man einmal die eigene Nabelschau aufgibt, ist es nicht sehr schwierig, in Südostasien die offensichtlichen Tatsachen auszumachen. Vietnam stellt eine eigenständige, unabhängige und dynamische Form des Kommunismus an der Grenze zu China dar. Das paßt China durchaus nicht ins Konzept. Die Volksrepublik erhebt Anspruch auf vietnamesische Küstengewässer, was zur Folge hätte, daß Schiffe auf dem Weg von Saigon nach Haiphong durch chinesische Territorialgewässer fahren müßten. China hat Pol Pots unprovozierten Angriff unterstützt. China reklamiert „eine Million und mehrere Hunderttausend" ethnischer Chinesen in Vietnam 
(um die chinesische Mathematik zu zitieren!) als seine Staatsbürger. Eine der Ursachen des gegenwärtigen Kriegszustandes liegt darin, daß Hanoi darauf bestanden hat, die ethnischen Chinesen als vietnamesische Staatsbürger aufzufassen. Wogegen die Forderungen Pekings darauf hinausliefen, Cholon, das Chinesenviertel von Ho Chi Minh-Stadt, nachgerade zum extraterritorialen Gebiet zu machen, dessen Besitz und Handelsunternehmen nach Artikel 54 der neuen chinesischen Verfassung dem direkten Schutz Pekings unterstellt gewesen wäre. Dieser Artikel wurde übrigens genau in dem Monat verkündet, in dem Hanoi ein Gesetz über die Nationalisierung der Handelsunternehmen in Cholon erließ. Abgesehen von dieser erstaunlichen Serie politischer Forderungen verkörpert China auch eine weniger demokratische Form des Kommunismus. Ich unterstelle nicht, daß ein Land wie Vietnam, das vorwiegend bäuerlich ist und dazu von Hunger bedroht, kurz davor stehen könnte, seiner arbeitenden Klasse Verhältnisse einer unbeschränkten politischen Freiheit zu bieten. Aber daß es in der vietnamesischen KP keine Säuberungen gegeben hat, zeigt doch an, daß in ihr eine menschliche und kollektive Disziplin herrscht, die der chinesischen überlegen ist. Auf Südostasien bezogen entspricht daher Vietnam eher der CSSR gegenüber einer Volksrepublik China, die eher in der Rolle der Sowjetunion auftritt. Der Sozialismus ist in Vietnam weiter fortgeschritten und die nationale und politische Unabhängigkeit des Landes ist eine Errungenschaft, die Sozialisten in der ganzen Welt unbedingt bewahren helfen sollten. Der Unterschied zwischen der Invasion in der CSSR und der chinesischen Invasion in Vietnam ist der, daß die Vietnamesen in der Lage waren, an allen Fronten zurückzuschlagen; und dies vor allem aufgrund ihrer inneren Stärke, aber auch aufgrund der wichtigen Hilfe, die sie vom ältesten sozialistischen Staat erhalten haben. Dies mag paradox klingen, aber - um es noch einmal zu sagen - wenn die Sozialisten in Europa, und besonders die größeren kommunistischen Parteien in Ost- wie in Westeuropa, sich nicht auf diese gar nicht so verblüffende Umkehrung ihrer europäischen Maßstäbe einstellen, werden sie es sein, die eine brutale sozialistische „Realpolitik" vertreten - und die in China eine noch stalinistischere Form von Politik unterstützen. 